\title{
CARTESIAN SLIDING PD CONTROL OF ROBOT MANIPULATORS FOR TRACKING IN FINITE TIME: THEORY AND EXPERIMENTS
}

\author{
DomingueZ Ramirez, O.A.; PARRA VegA, V.; DiaZ Montiel, \\ M.G.; Pozas CARDENAS, M.J. \& HERnANDEZ GOMEZ, R.A.
}

Abstract: For a class of robot arms, the PD controller plus and PID controller can not render asymptotic stability for tracking tasks. In this work a cartesian control system is proposed which guarantees robust tracking in finite time based on time base generator for uncertain robot arms, to reach this goal we proposed a nonlinear control based on second order sliding modes and we present the design and experimental results in a robot without gravity component and small friction parameters

Key words: Robot manipulator, adaptive control, sliding mode control, finite time convergence, time base generator
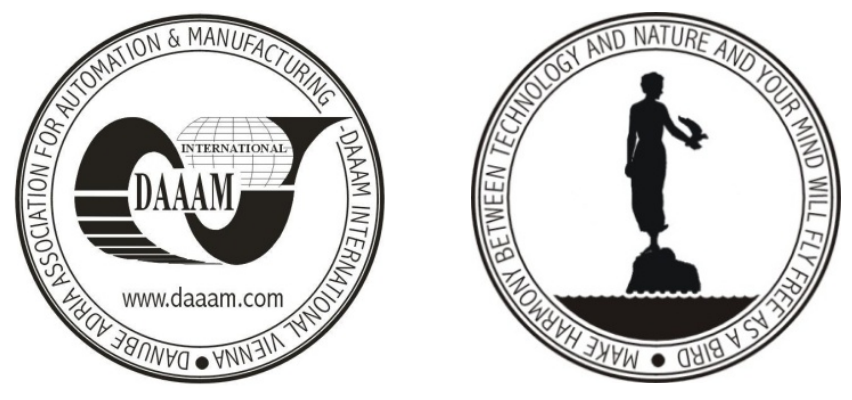

Authors' data: Ph.D. M.Sc. Domínguez Ramírez, O[mar] A[rturo]*; Prof. Ph.D. M.Sc. Parra Vega, V[icente]**; M.Sc. Eng Díaz Montiel, M[ayen] G[icela]*; M.Sc. Pozas Cárdenas, M[ariano] J[avier]*; Ph.D. M.Sc. Hernández Gómez, R[oberto] A[rmando]*;* Universidad Autónoma del Estado de Hidalgo, Instituto de Ciencias Básicas e Ingeniería, Área Académica de Computación, Centro de Investigación en Tecnologías de Información y Sistemas, Carretera Pachuca-Tulancingo Km 4.5 Ciudad Universitaria, Pachuca, Hidalgo, México, **Robotics and Advanced Manufacturing Division, Research Center for Advanced Studies Saltillo Campus CINVESTAV Carretera Saltillo-Monterrey, Km 13.5, Ramos Arizpe, Coah, 25900 México, email: omar@uaeh.edu.mx, vparra@cinvestav.mx, neyamgc@hotmail.com, mpozas@uaeh.edu.mx, armando@uaeh.edu.mx

This Publication has to be referred as: Dominguez Ramirez, $\mathrm{O}$ [mar] A[rturo]; Parra Vega, V[icente]; Diaz Montiel, M[ayen] G[icela]; Pozas Cardenas, M[ariano] J[avier] \& Hernandez Gomez, R[oberto] A[rmando] (2008). Cartesian Sliding PD Control of Robot Manipulators for Tracking in Finite Time: Theory and Experiments, Chapter 23 in DAAAM International Scientific Book 2008, pp. 257-272, B. Katalinic (Ed.), Published by DAAAM International, ISBN 978-3-901509-66-7, ISSN 1726-9687, Vienna, Austria

DOI: $10.2507 /$ daaam.scibook.2008.23 